\title{
Modelling and Simulation of AdHoc Network Traffic Based on Tactical Communication Environment
}

\author{
Lu Ying ${ }^{*}, 1$ Kang Feng-Ju ${ }^{2}$ and Zhong Lian-Jiong ${ }^{1}$ \\ ${ }^{I}$ Computer Science and Engineering College, Xi'an Technological University, Xi'an 710032, China \\ ${ }^{2}$ College of Marine Engineering, Northwestern Polytechnical University, Xi'an 710072, China
}

\begin{abstract}
Tactical communication network environment is different to the civil mobile adhoc network. It is of special connectivity, traffic flow characteristic and mobility. Its traffic flow model is the foundation for adhoc network designing, planning, controlling and managing. Each characteristic can be depicted correctly only when the model is accuracy. This thesis studied the traffic flow characteristics of the tactic adhoc network and putting forward an implementation method of creating traffic flow model. To solve the problem of loading traffic distribution model, it provides an address generating model using improved OPNET PDF probability editor named "PDF Destination Address model" which can send packets in accordance with traffic flow distribution proportionate relations matrix between each units. It can achieve traffic flow more quickly and effectively. Simulation experiment is operation in different parameters conditions and the results demonstrate that the proposed model of generating traffic flow between each units is effective. This method which can meet each unit's requirements of traffic flow size, intensity and orientation can be effectively applied in tactical adhoc communication network simulation and performance research.
\end{abstract}

Keywords: Adhoc, OPNET PDF, proportionate relations matrix, traffic flow.

\section{INTRODUCTION}

As the communication technology developing and battlefield operational requirements changing, the scale of tactic communication network and all kinds of applications get more and more complicated and diversified. Therefore, modeling and analyzing of its traffic flow has significant effect meaning. It is the foundation and premise of network analysis, protocol designing, network planning and management. So far, there is lacking of an integrity and effective studying method and system of traffic flow modeling for tactic communication network inside or outside of the country. Thus, a study method is needed, which is from the perspective of networking technology and actual characteristics of operations. Also, it combines with concurrent mathematical theories and tool selection that suitable for its traffic characteristic. Basing on this, a method system is constructed, which includes network traffic characteristics analysis, traffic modeling, protocol designing and network performance evaluation. During the past network simulation process, the size and strength of the traffic flow amount between each terminal and command post is just simply fixed setup.

It is barely mention about the intensity distribution of the traffic flow is following the proportional distribution of the relationships or requirements of user units and ration calculation during traffic flow modeling and simulation process, in order to inspect and evaluate the communication capability of the whole system. This paper conducted studying and analysis of traffic amount characteristics and suitable statistical property model of the audio, data, video operation of the tactic communication network in the first place. Then, in allusion to the issue of loading traffic flow distribution model on the traffic stream statistical model and the way of accomplishing, a PDF Destination Address model based on OPNET PDF editor is brought up. Using this method to load flow distribution model conforms to the actual relations of distribution of traffic proportion. It can meet each command unit's requirements of flow intensity, size and direction effectively. This paper conducted simulations of tactic communication network based on the study mentioned above.

\section{CONSTRUCTION OF NETWORK TRAFFIC STATISTICAL MODEL}

Conducting theoretical study about tactic communication network's performance and protocols requires constructing traffic model that is close to the statistical characteristics of actual traffic stream for simulating input. Tactic communication network is a kind of packet radio networks, which takes burst data traffic as principle traffic. Its supporting communication operation including position report, real time voice, IP message transmission, video, etc. All these operations possess randomness and burstiness [1]. Self-similar model shows better long rang dependency and burstiness in relatively wide time scale, which is more close to the actual traffic characteristics [2]. Moreover, wartime communication always shows status like, Good/Bad of connection status; Move/Stop of battle field mobile user status; Have/Not Have of battle information status and so on. Large amount of these ON/OFF data source stacking presents Noah effect [3], which cause traffics show self- 


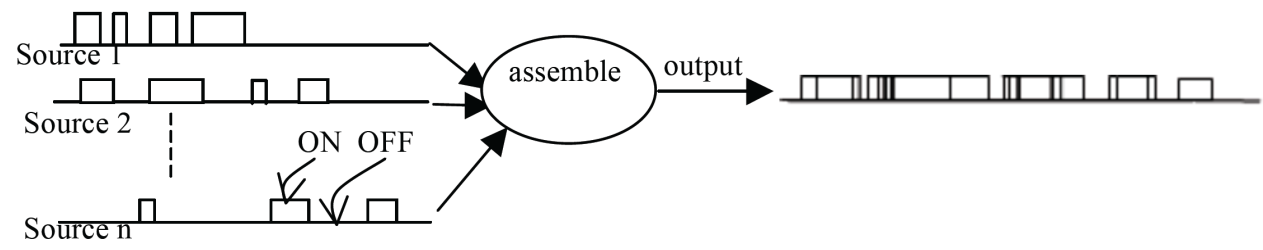

Fig. (1). Assembled ON/OFF sourse model.

similarity on mathematics. Therefore, this paper adopted self-similar model for simulation of audio, data, video traffics in tactic communication network. Also, it used statistical prospects to make network performance approach mathematics outcomes.

Self-similar traffic usually is generated by using heavytailed ON/OFF source stacking model. This is a modelling method basing on the physical significance, which is easy to control and has good self-similar effect [4]. When the number of data sources approaching infinite, the traffic generated approaches self-similar gradually. As Fig. (1), each source switching between $\mathrm{ON}$ and OFF. When it is at ON status, the traffic transmits data with constant speed. When it is at OFF status, it stops. The time duration of ON and OFF is independent and meets heavy-tailed distribution Pareto $(\alpha, \mathrm{k})$.

$\alpha$ is shape parameter, $\mathrm{k}>0$ is positional parameter, they represent the minimum of random variables. Self-similar degree is determined by shape parameter, $\alpha$. The relationship between $\alpha$ and $H$ is: $H=(3-\alpha) / 2$, the smaller the $\alpha(1<\alpha<2)$, the larger the self-similar degree. Because the traffic generated by ON/OFF sources of single Pareto distribution is just asymptotic, requiring infinite data sources stacking in order to achieve self-similarity. It is difficult to accomplish in reality [5]. Using OPNET technology during simulating, self-similar traffic is generated through improving source module in terminal node layer model and constructing ON/OFF source groups with heavy-tailed distributed duration time as many as possible. Please see reference $[6,7]$ for detailed process and method. Three kinds of traffics can be generated through configuring the message sending sources and adjust transmitting speed of the source of network node model according to the specific requirements during simulation.

\section{CONSTRUCTION OF TRAFFIC DISTRIBUTION MODEL}

After getting traffic statistical characteristics model, it requires battlefield communication requirements and the business relations between each command post to get the traffic distribution model which describes the size, intensity, directions of information flow between command posts and users within the system. According to tactic communication network's characteristics, its business traffic direction has fixed regular patten and the commanding and cooperation relations between command posts are relatively invariant. Thus, it is possible to get the degree of closeness which can be used as the proration allocation reference of traffic through analysis and forecasting [8]. Traffic size and direction will be allocated reasonably according to its requirements in actual simulation process.

This paper defines the product of traffic proportionate relationships matrix multiply by the average business traffic intensity of this kind of traffic in the network as the values of traffic flow distribution matrix between command posts.

$F_{i j}=S_{i j} \times P$

In the above formula, $F_{i j}$ represents the traffic distribution matrix of certain business, $S_{i j}$ represents proportionate relations matrix, $P$ represents the average business traffic intensity of this kind of business in the network. The proportionate relations matrix is shown as Table $\mathbf{1}$.

From formula (1) and the table above, we get that the calculation of traffic flow of Company 0 should be Company $_{0}=$ $P\left(S_{00}+S_{01}+S_{02}+S_{03}+S_{04}\right)$. Using the same method, we can get the traffic sizes of all of other command posts.

In conclusion, network business traffic flow distribution matrix can be got by knowing user distribution of all kinds of business, the traffic proportionate distribution relations between command posts, and the average traffic intensity data of whole users in the network.

\section{A METHOD OF LOADING AND ACCOMPLISH TRAFFIC DISTRIBUTION MATRIX}

How to load traffic distribution matrix rapidly and precisely in the simulation model is a question worthy of consideration during modeling. This paper adopted OPNET

Table 1. Traffic flow distribution matrix between command posts.

\begin{tabular}{|c|c|c|c|c|c|}
\hline Unit & Company $_{0}$ & Company $_{1}$ & Company $_{2}$ & Company $_{3}$ & Company $_{4}$ \\
\hline \hline Company $_{0}$ & $S_{00}=1.0$ & $S_{01}=1.0$ & $S_{02}=1.0$ & $S_{03}=1.0$ & $S_{04}=1.0$ \\
\hline Company $_{1}$ & $S_{10}=1.0$ & $S_{11}=0.8$ & $S_{12}=0.8$ & $S_{13}=0.5$ & $S_{14}=0.9$ \\
\hline Company $_{2}$ & $S_{20}=1.0$ & $S_{21}=0.8$ & $S_{22}=1.2$ & $S_{33}=1.2$ & $S_{34}=0.3$ \\
\hline Company $_{3}$ & $S_{30}=1.0$ & $S_{31}=0.5$ & $S_{32}=0.7$ & $S_{43}=0.6$ & $S_{42}=0.3$ \\
\hline Company $_{4}$ & $S_{40}=1.0$ & $S_{41}=0.9$ & $S_{4}$ & \\
\hline
\end{tabular}


technology as the tool for simulation and modeling. The PDF Editor provided by OPNET can be used for creating, editing and reading probability density function $[9,10]$. During the process of in-depth understanding, studying and analyzing OPNET, this paper put up a traffic proportionate allocating method basing on PDF Editor to achieve traffic allocation and generation of each unit during simulation. Its principle is describing and defining it by calculating the probability of a node sending packets to other nodes according to the relations of traffic distribution and combining with using applications to control the PDF Editor. During defining, associating PDF Editor's horizontal axis as the MAC address of destination node and vertical axis as the weight of sending packets to the destination. Then, loading this PDF probability distribution function in wlan_mac_interface module; using the calculated results as the destination addresses of the generated packets. By using this method to achieve correspondence of traffic proportionate distribution matrix and loading and processing on mobile nodes.

The address of the packets generated by nodes can only be set as fixed node MAC or set as Random, which cannot control the traffic directions and probabilities according to the proportions in OPNET. This paper introduced the address generating model which can send packets in accordance with traffic distribution proportions. Because this model achieves the goal through flexibly using the OPNET PDF Editor and combining with relevant code, this paper called it PDF Destination Address model.

Fig. (2) is the editing UI of PDF file. Horizontal axis represents for the selection value of function, $\mathrm{F}(\mathrm{x})$, vertical axis represents the probability or weight of the selection value, $\mathrm{P}(\mathrm{x})$. In the algorithm, it defines the horizontal axis as the MAC address of the whole network and the vertical axis as the probability of sending to this MAC address. According to the relations of traffic proportions between command posts and traffic proportional relation within the command posts from Table 1, it creates PDF file of each corresponding command posts, as Figs. $(\mathbf{3}, \mathbf{4})$, assigning to the Destination Address property of relevant nodes. Fig. (3a) is the PDF file of Company 0 , horizontal axis as the MAC address of the destination and the vertical axis as the weight of sending to this MAC address. The one with weight value is the valid destination node.

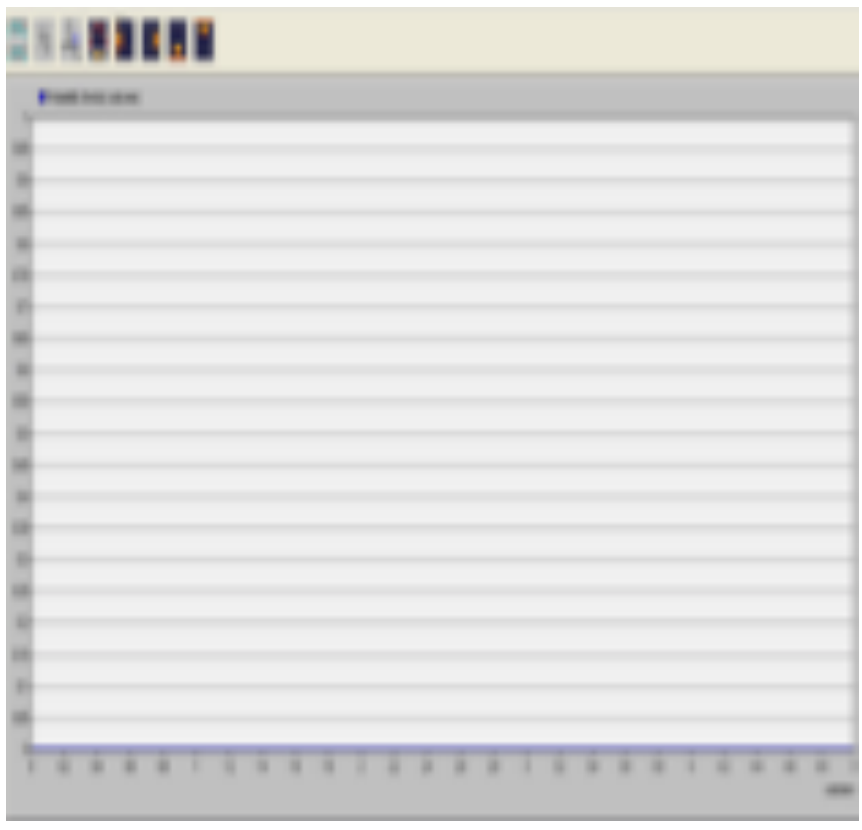

Fig. (2). PDF editor.

During the achieving process, it requires to set the nodes' destination address properties of Company 0 as PDF file, military_net_company0.pd. Also, it needs to set PDF files of Company 1-4 accordingly to eventually accomplish the

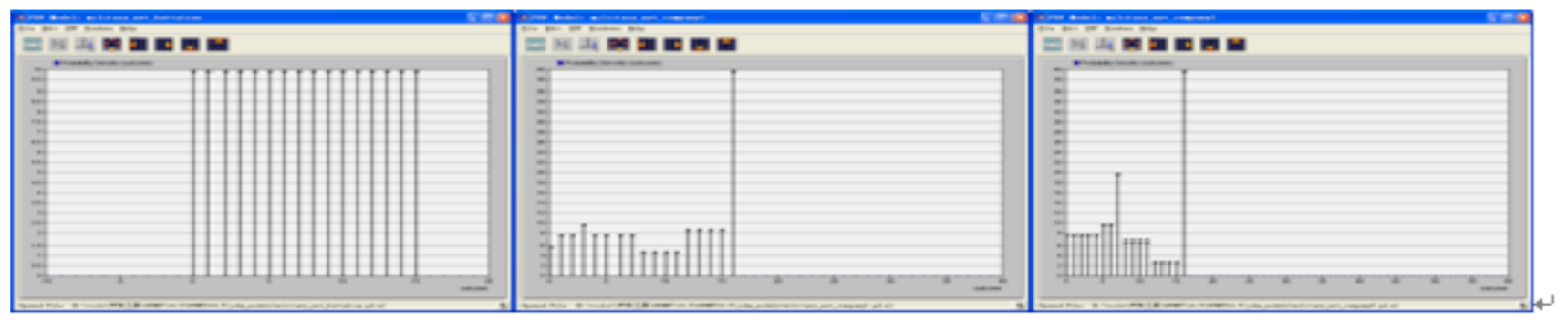

(a) military_net_companyo.pd (b)military_net_company1.pd

(c)military_net_company2.pd*

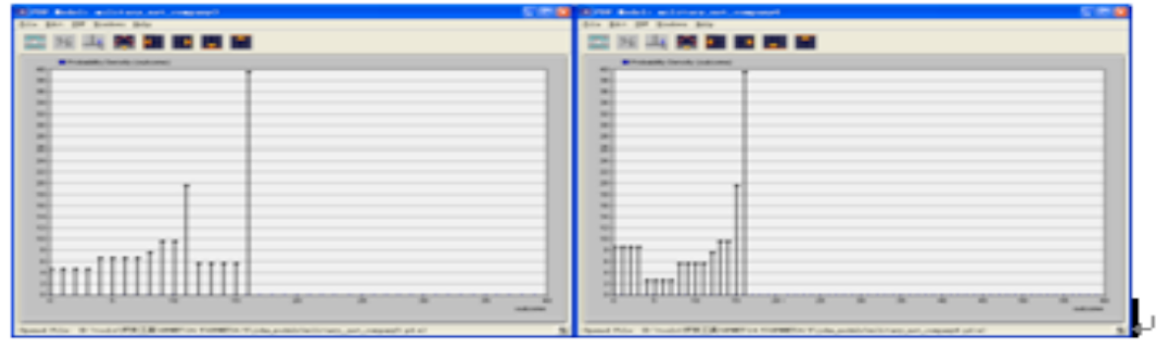

(d)militarv net companv3.pd

(e)militarv net combanv4.pd

Fig. (3). The PDF files of command. 


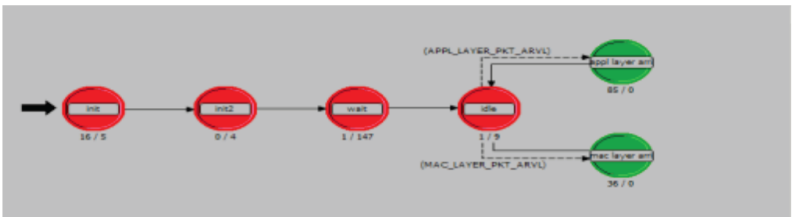

\begin{tabular}{|c|c|c|c|}
\hline At tribute Kane & Group Type & Units Default Value & Tags \\
\hline stination Address & intege & Randon & \\
\hline $\begin{array}{l}\text { ppe of Service } \\
\text { estPdf }\end{array}$ & $\begin{array}{l}\text { intege } \\
\text { string }\end{array}$ & Best Effort (0) & \\
\hline
\end{tabular}

(a) wlan_mac_intf process moduel (b) wlan_mac_intf model properties configuration

Fig. (4). The configurations of relevant parameters and properties.

loading of traffic distribution matrix in simulation model. While the application is running, the selected values of PDF files are used as references of the proportions of sending data to the destination. As the result, the traffic intensity distribution of each command post is controlled precisely.

This method requires some configurations of relevant parameters and properties in OPNET during achieving:

1) Configuration parameters can be adjusted in interface $\rightarrow$ Model Attributes in wlan_mac intf process module of node model to support PDF destination address model, see Fig. (4). A DesPdf customized property is designed and added in model property panel.

2) Further editing the Destination Address properties. Its original parameter' $s$ read values have only Random(-2, random), Broadcast(-1, broadcast) and fixed MAC addresses. Now, it adds a customized DestPdf type, read value is -3 . When the application gets a variable of -3 during running, it means destination address allocating according to the PDF file defined by this paper. Furthermore, adding a private parameter is needed, which is used for loading the PDF file's name.

3) Configuring the values of Destination Address parameters on the selected nodes as the model designed by this paper, DestPdf, and configuring value of DestPdf as the loading PDF file name.

4) When achieving, it also requires giving the relevant controlling code in wlan_mac interface and wait state of process model, to load pdf file and generates pdf handles. After process model receiving the packets generated by "Source" origin, it triggers APPL_LAYER_PKT_ARVL events, loading relevant $\mathrm{PDF}$, gets corresponding destination address.

\section{SIMULATION AND EXPERIMENTAL ANALYSIS IN TACTIC COMMUNICATION NETWORK}

Construct a double-layer distributed communication network containing 4 company level sub-net as the bottom layer and a battalion level sub-net. The number of nodes within each company level sub-net is 4 ; the number of inputting nodes of battalion level sub-net is also 4. Simulating nodes adopts DCF mechanism of IEEE802.11 standards to achieve the competing input of channels. The corresponding organizational structure of this network model is shown as Fig. (5), company_subnet_1 to company_subnet_4 is the company level sub-nets and company_subnet_0 is the battalion level sub-net.

The "Source" origin module of node model loads three kinds of traffics, audio and data and video, in accordance to the business needs. In facts, only two kinds of business traffics are required to be configured from the perspectives of statistical characteristics of each business kinds, data and video business traffic which possess self-similarity and Markov audio business traffic. Business traffic is generated through setting the packet sending sources of the "Source" module of node model proportionately. As Fig. (6) shown, data and video traffic Source team is responsible for generating the self-similar traffic; audio Source team is responsible for generating Markov business traffic.

Next, it requires loading corresponding PDF file in wlan_mac_intf module of node model in order to achieve correspondence of traffic allocation between command posts. Here has loaded 5 PDF files, as shown on Fig. (3), for each node of 5 sub-nets respectively.

Configuring the averages traffic intensity of audio, data and video traffic as shown on Table 2 during simulation, P1, P2 and P3 is stand for the traffic intensity in descending

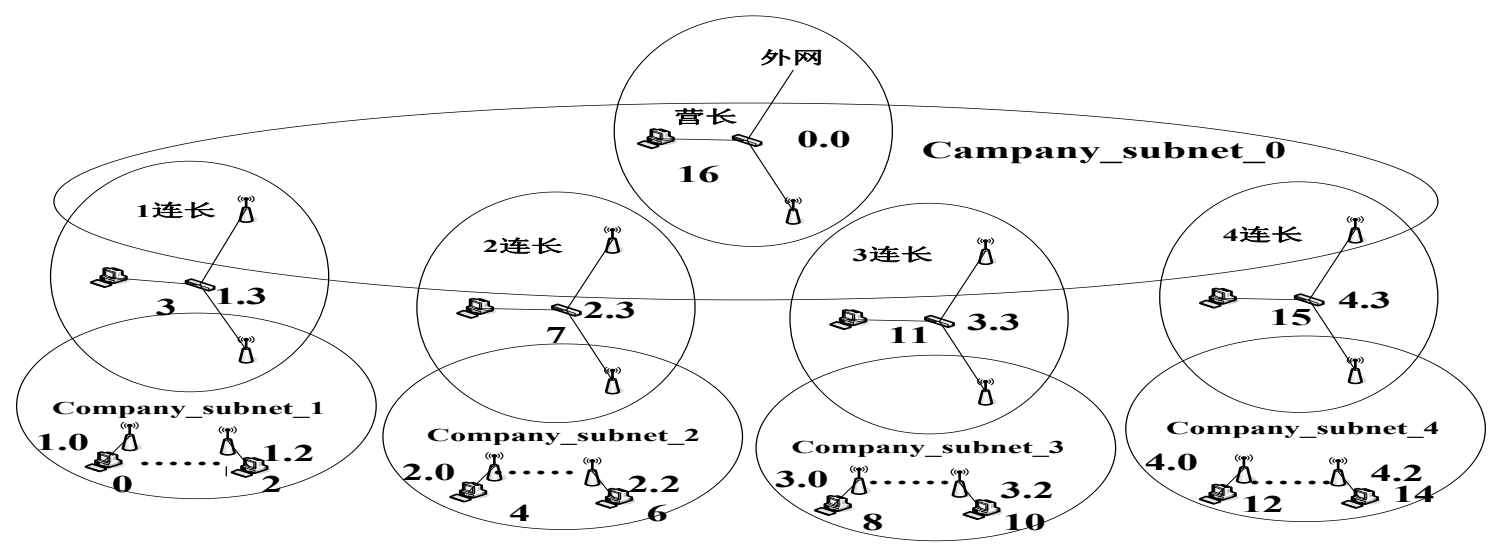

Fig. (5). Organizational structure of the network. 


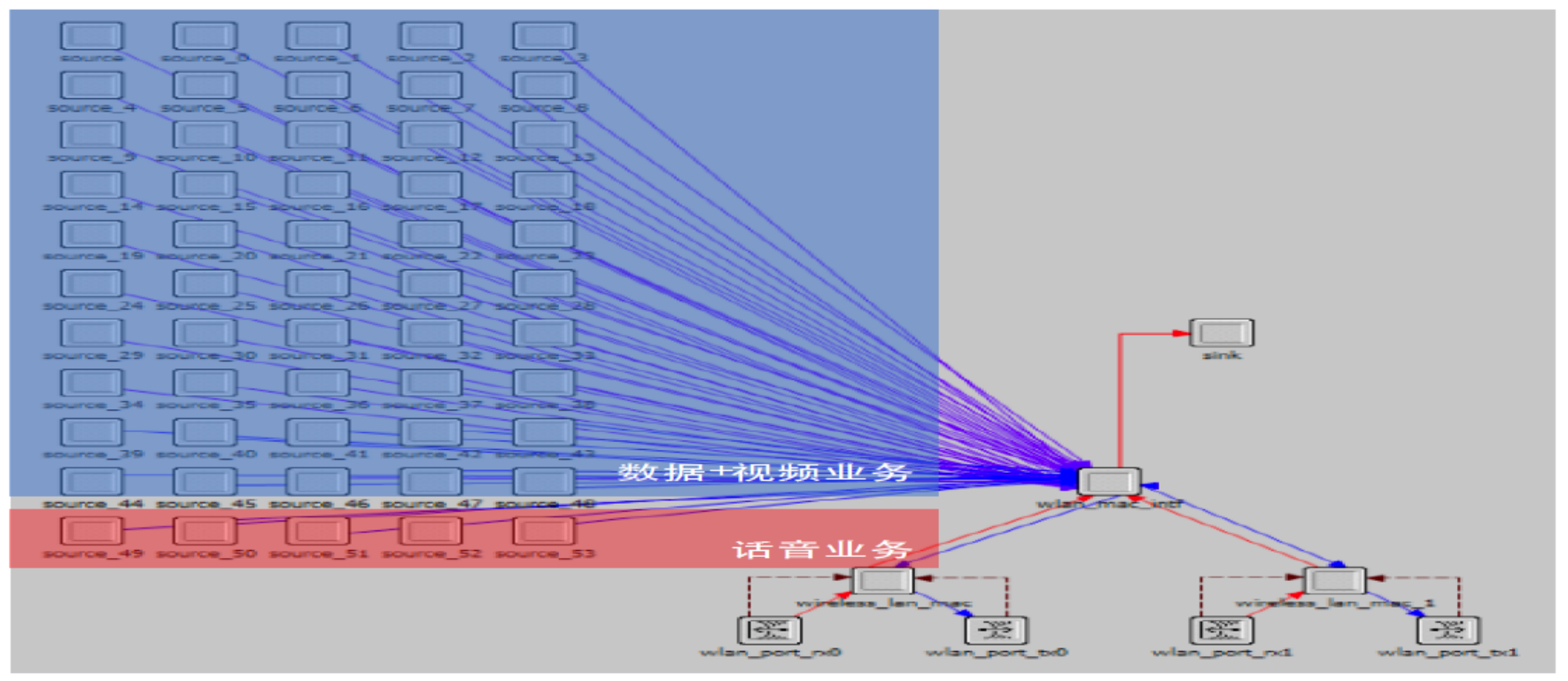

Fig. (6). The "Source" origin module of node model.

Table 2. Traffic intensity of three business.

\begin{tabular}{|c|c|c|c|}
\hline The Proportion of Traffic (\%) & P1 Traffic Intensity (bit/s) & P2 Traffic Intensity (bit/s) & P3 Traffic Intensity (bit/s) \\
\hline \hline audio (9\%) & $\mathrm{P}_{11}=3943.07$ & $\mathrm{P}_{21}=2303.17$ & $\mathrm{P}_{31}=594.425$ \\
\hline data (87\%) & $\mathrm{P}_{12}=38609.3$ & $\mathrm{P}_{22}=22551.9$ & $\mathrm{P}_{32}=5820.42$ \\
\hline video (4\%) & $\mathrm{P}_{13}=1588.17$ & $\mathrm{P}_{23}=927.664$ & $\mathrm{P}_{33}=239.420$ \\
\hline
\end{tabular}

order, parameters of traffic generation is shown as Table 3. For simplifying the simulation, the experiment used 1 proportionate distribution matrix (Table 1) to show the proportions of 3 kinds of traffics.

\subsection{Experiment: the Performance of Network Under Different Traffic Intensities and Bandwidth}

Simulation was taken place when the traffic intensities are P1, P2 and P3 and the bandwidths are $512 \mathrm{k} / 256 \mathrm{k} / 128 \mathrm{kbit}$. It selected network throughput, lag, packet loss rate, successful sending rate and other performance indexes as the simulating analyzing parameters. Successful sending rate means the ratio of all packets that are successfully sent and total amount of packets generated by source nodes, which reflects the effectiveness of data transmission. Collecting simulation statistical data, we got the following curves of simulating results, as shown on Fig. (7).

As Fig. (7) shown, when bandwidth is $512 \mathrm{kbit}$, each network performance indexes changes as the simulation carry forward. There into, horizontal axis represents for simulating time (s), vertical axis represents network load, throughput, end to end time lag, packet loss rate respectively.

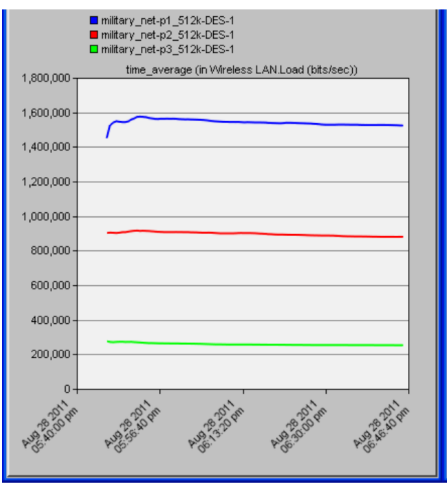

(a) load

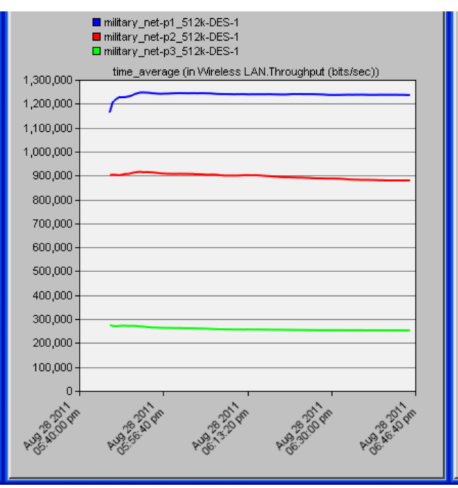

(b) throughput

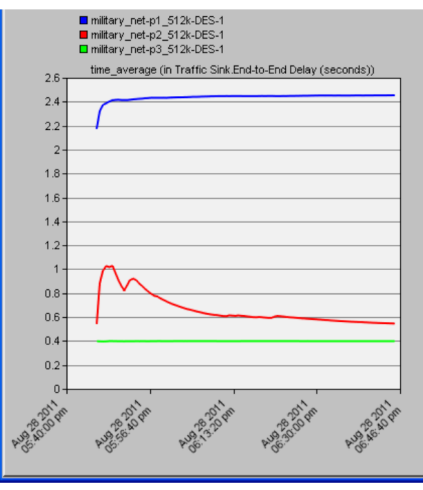

(c) end to end time lag

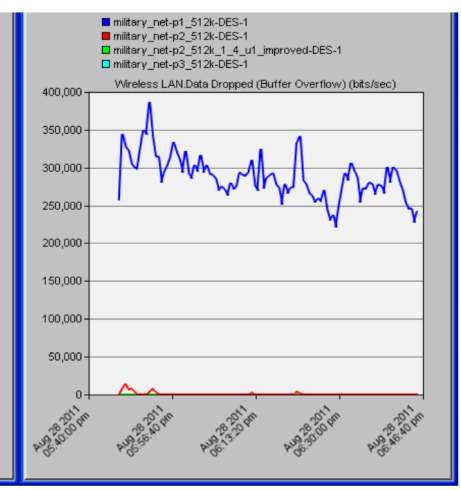

(d) packet loss rate

Fig. (7). The comparison of each performance index under 512kbit bandwidth and $\mathrm{P} 1 \backslash \mathrm{P} 2 \backslash \mathrm{P} 3$ traffic strength. 
From Fig. (7a), using P1 as heavy load, the result of packet loss is more serious; P2 is the medium load and P3 is the light load. Network throughput rises as the traffic intensity increases progressively. It reaches max under P1 intensity, and under P3 is the min. The network almost has no loss packet under $\mathrm{P} 2$ and $\mathrm{P} 3$ intensities.

Table 3. Parameter configuration table of traffic flow model under P2 intensity.

\begin{tabular}{|c|c|c|}
\hline \multirow{4}{*}{$\begin{array}{c}\text { data } \\
\text { traffic }\end{array}$} & ON stats time distribute & Pareto(2,1.6) \\
\cline { 2 - 3 } & OFF stats time distribute & Pareto(2,1.6) \\
\cline { 2 - 3 } & packet rate & $22 \mathrm{kbit} / \mathrm{s}$ \\
\hline \multirow{4}{*}{$\begin{array}{c}\text { audio } \\
\text { traffic }\end{array}$} & length of data packet & exponential distribution of 512byte \\
\cline { 2 - 3 } & ON stats time distribute & Expenontial (0.15) \\
\cline { 2 - 4 } & OFF stats time distribute & Expenontial (1.20) \\
\cline { 2 - 4 } & length of data packet & Expenontial (1.00) \\
\hline \multirow{3}{*}{$\begin{array}{l}\text { video } \\
\text { traffic }\end{array}$} & ON stats time distribute & Pareto(2,1.6) \\
\cline { 2 - 3 } & OFF stats time distribute & Pareto(2,1.6) \\
\cline { 2 - 4 } & packet rate & 22kbit/s \\
\cline { 2 - 4 } & length of data packet & exponential distribution of 512byte \\
\hline
\end{tabular}

Table 4 shows the statistical results of data successful sending rate, calculated simultaneously, under 3 different bandwidths and intensities, P1, P2 and P3. Combining Fig. (7) and Table 4, when it is under 512kbit and P1, successful sending rate is only $67.45 \%$. It reaches $97.2 \%$ under P2. And it reaches $99.3 \%$ under $\mathrm{P} 3$. When average traffic intensity reaches $\mathrm{P} 1$, time lag rises faster and successful sending rate is low, which is below the minimum requirements of communication indexes during the battle processes (receiving rate of packets must not below 90\%). But under P2 and P3 intensities, the statistical results of simulation meet the expectations. With $128 \mathrm{kbit}$ and $256 \mathrm{kbit}$ bandwidths, statistical results of simulation under P1 and P2 intensities exceed the scopes of technical indexes, do not meet the requirements.
From the result above, with the double-layering tactic communication network and traffic flow parameter set by this paper, the network will be relatively in a ideal status when intensity is P2 and bandwidth is $512 \mathrm{kbit}$.

Within the battle environment that moving fast, high bandwidth can reduce packet loss or performance loss, reducing transmitting errors. Conversely, as the bandwidth decreases, the time-lag of message sending will be longer, which will lead to network jam and increase the packet losses. Also, frequent moving of battle field nodes will increase the probability of packet loss and resending, which will further increase the time-lag and worse the network performance. Battle field network bandwidth is often limited by many reasons. Therefore, enlarge the buffer or other network resources can be considered. Or the encoding technologies with higher compression ratio can be adopted when the quality of audio and video is ensured from the perspectives of traffic, so that the resources acquired are ensured with limited bandwidth.

\section{CONCLUSIONS}

This paper conducted analysis and study of flow modeling technology of tactic communication network. And it confirmed the statistical property model that is needed for traffic flow modeling. It also brought up an accomplishing algorithm of flow distribution model based on PDF editor, which can effectively solve the issue of loading flow distribution model on traffic stream statistical model. The flow modeling process and methods brought up by this paper has significant value for tactic communication network with large volume of data transmission. It can provide references for network planning and designing.

\section{CONFLICT OF INTEREST}

The authors confirm that this article content has no conflicts of interest.

\section{ACKNOWLEDGEMENTS}

This work is supported by the Key Project of Guangxi Social Sciences, China (No. gxsk201424), the Education

Table 4. Statistical results of data successful sending rate under P1, P2 and P3 intensities and 3 different bandwidths.

\begin{tabular}{|c|c|c|c|c|c|c|}
\hline $\begin{array}{c}\text { Traffic } \\
\text { Intensity }\end{array}$ & $\begin{array}{c}\text { Bandwidth } \\
\text { (bit) }\end{array}$ & $\begin{array}{c}\text { Total Number of } \\
\text { Data Packets }\end{array}$ & $\begin{array}{c}\text { Length of Data } \\
\text { Packet (B) }\end{array}$ & $\begin{array}{c}\text { Data Amount of } \\
\text { Generation (bit/s) }\end{array}$ & $\begin{array}{c}\text { Data Amount of } \\
\text { Received (bit/s) }\end{array}$ & $\begin{array}{c}\text { Successful } \\
\text { Sending Rate }\end{array}$ \\
\hline \hline \multirow{3}{*}{ P1 } & $512 \mathrm{k}$ & 752396 & 528 & 882811.307 & 595480.747 & $67.45 \%$ \\
\cline { 2 - 7 } & $256 \mathrm{k}$ & 762316 & 528 & 894450.773 & 388238.400 & $43.4 \%$ \\
\cline { 2 - 7 } & $128 \mathrm{k}$ & 771508 & 528 & 905236.053 & 237046.187 & $26.2 \%$ \\
\hline \multirow{3}{*}{ P2 } & $512 \mathrm{k}$ & 396872 & 528 & 479020.576 & 465608.000 & $97.2 \%$ \\
\cline { 2 - 7 } & $256 \mathrm{k}$ & 408947 & 528 & 479831.147 & 311754.667 & $64.97 \%$ \\
\hline \multirow{3}{*}{ P3 } & $128 \mathrm{k}$ & 405760 & 528 & 476091.733 & 201367.467 & $42.29 \%$ \\
\cline { 2 - 7 } & $512 \mathrm{k}$ & 113425 & 528 & 134147.956 & 133074.773 & $99.3 \%$ \\
\hline
\end{tabular}


Science fund of the Education Department of Guangxi, China (No.2014JGA268), and Guangxi Office for Education Sciences Planning, China (No.2013C108).

\section{REFERENCES}

[1] C. Min, OPNET Network Simulation, Tsinghua University Press, Beijing, 2004.

[2] W. E. Leland, M. S. Taqqu, W. Willinger, and D. V. Wilson, "On the self-similar nature of Ethernet traffic", ACM SIGCOMM Computer Communication Review, 1993, pp. 183-193.

[3] W. Yang-bo, "Backoff algorithm suitable for burst traffic character of tactical adhoc network," Computer Simulation, vol. 4, p. 35, 2012.

[4] Y. Lu, F.-J. Kang, L.-J. Zhong, and X.-Y. Liang, "The research of generation model of tactical subnet flow and analysis of network performance," Huoli yu Zhihui Kongzhi, vol. 36, 2011.
[5] L. Hao. "Approach research for adhesion coefficient and its application", Information Command Control System \& Simulation Technology, pp. 49-51, 2004.

[6] V. Paxson and S. Floyd, "Wide area traffic: the failure of Poisson modeling," IEEE/ACM Transactions on Networking (ToN), vol. 3, pp. 226-244, 1995.

[7] Q. Wang and T. Zhang, "Source traffic modeling in wireless sensor networks for target tracking," In: Proceedings of the $5^{\text {th }}$ ACM symposium on Performance evaluation of wireless ad hoc, sensor, and ubiquitous networks, pp. 96-100, 2008.

[8] W. Stallings, High Speed Networks, TCP/IP and ATM Design Principles, Upper Saddle River, NJ, Prentice Hall, Inc., 1998.

[9] L.-M. Zhang, "Research on propagation nature of self-similarity of traffic in network," Jisuanji Gongcheng yu Yingyong (Computer Engineering and Applications), vol. 42, pp. 141-144, 2007.

Received: September 16, 2014

(C) Ying et al.; Licensee Bentham Open.

This is an open access article licensed under the terms of the (https://creativecommons.org/licenses/by/4.0/legalcode), which permits unrestricted, non-commercial use, distribution and reproduction in any medium, provided the work is properly cited. 